Pacific Journal of Mathematics

COUNTEREXAMPLE IN THE THEORY OF CONTINUOUS 


\title{
COUNTEREXAMPLE IN THE THEORY OF CONTINUOUS FUNCTIONS ON TOPOLOGICAL GROUPS
}

\author{
P. Milnes and J. S. Pym
}

If $G$ is a topological group and $\tau$ is the topology on $C(G)$ of pointwise convergence on $G$, a function space $\mathcal{M}(G)$ of almost periodic type is defined by $\mathcal{M}(G)=\left\{f \in C(G) \mid\left\{\boldsymbol{r}_{s} f \mid s \in G\right\}\right.$ is relatively $\tau$-compact $\}$. Generalizing results of $\mathbf{T}$. Mitchell, $\mathbf{C}$. $\mathbf{R}$. Rao, and P. Milnes, we show here that $\mathcal{M}(G)$ is just the left uniformly continuous subspace, $L U C(G)$, of $C(G)$ for groups satisfying a completeness condition and give an example on the rational numbers which shows that some completeness condition is necessary for this conclusion to hold. The example also shows that, if $G$ is a dense subgroup of a topological group $G^{\prime}$, functions in $\mathscr{M}(G)$ (which are known always to extend to functions in $C\left(G^{\prime}\right)$ ) need not extend to functions in $\mathcal{M}\left(G^{\prime}\right)$; this result is at variance with what happens in the case of the familiar almost periodic or weakly almost periodic functions, where a function always extends to a function of the same type.

(The conclusions of the theorems of this paper hold in more general settings than have been described above.)

1. Generalities. Let $G$ be a topological group and let $\tau$ denote the topology on $C(G)$ of pointwise convergence on $G$. We define a subspace $\mathcal{M}(G)$ of $C(G)$ on $G$ by $\mathcal{M}(G)=$ $\left\{f \in C(G) \mid\left\{\boldsymbol{r}_{s} f \mid s \in G\right\}\right.$ is relatively $\tau$-compact $\}$. (Here $\boldsymbol{r}_{\mathrm{s}} f$ is the right translate of $f$ by $s, \boldsymbol{r}_{\mathrm{s}} f(t)=f(t s)$ for all $t \in G$.) If, in this definition, $\tau$ is replaced by the norm, resp. weak, topology of $C(G)$, one gets the more familiar almost periodic, resp. weakly almost periodic, subspace of $C(G)$. The space $M(G)$ was introduced in a different way by Mitchell [6] in the more general setting of semitopological semigroups. The following characterizations of $\mathcal{M}(G)$ (which also hold in this more general setting) are due to Mitchell [6], Baker and Butcher [1], and the first of the present authors [3]. More definitions and comments follow the statement of the theorem.

THEOREM 1. LMC $(G)$ is the largest left $m$-introverted subspace of $C(G$.$) . Also, f \in L M C(G)$ if and only if

(i) the function $s \rightarrow x\left(l_{s} f\right)$ is in $C(G)$ for all $x \in \beta G$. If $G$ is also a $k$-space, then $f \in L M C(G)$ if and only if one (hence all) of the following conditions holds: 
(ii) $\left\{l_{s} f \mid s \in K\right\}$ is weakly compact in $C(G)$ for all compact $K \subset G$.

(iii) $\left\{l_{s} f \mid s \in K\right\}$ is $\sigma(C(G), \beta G)$-compact in $C(G)$ for all compact $K \subset G$.

(iv) if the iterated limits $\lim _{n} \lim _{m} f\left(s_{m} t_{n}\right)$ and $\lim _{m} \lim _{n} f\left(s_{m} t_{n}\right)$ both exist, where $\left\{t_{n}\right\}$ and $\left\{s_{m}\right\}$ are sequences in $G$ with $\left\{s_{m}\right\}$ relatively compact, then they are equal.

Here $l_{s} f(t)=f(s t)$ for $s, t \in G$, and a subspace $X$ of $C(G)$ is called left $m$-introverted if the function $s \rightarrow x\left(l_{s} f\right)$ is in $X$ whenever $f \in X$ and $x \in \beta G ; \beta G$ is the spectrum of $C(G)$, which we regard as a subset of $C(G)^{*}$. A topological space $Y$ is called a $k$-space if every subset $A$ of $Y$, for which $A \cap K$ is closed for every compact $K \subset Y$, is necessarily closed. The $k$-spaces include spaces that are locally compact or first countable.

It is an easy exercise to show that (i) and (iii) are equivalent (in the $k$-space setting). The equivalence of (ii), (iii), and (iv) is proved using results of Grothendieck [2] and (iv) bears a striking similarity to Grothendieck's characterization of the weakly almost periodic functions [2; Proposition 7]. Another easy exercise (like the one showing (i) and (iii) are equivalent) involving (ii) shows that, in the $k$-space setting, $f \in M(G)$ if and only if the function $s \rightarrow x\left(l_{s} f\right)$ is in $C(G)$ for all $x \in C(G)^{*}$; this generalizes Mitchell's result (a), §5, of [6] and Proposition 4.2 in [3].

One of the hypotheses in the theorem which follows is a completeness assumption. We do not define it explicitly, but remark that locally compact spaces and complete metric spaces are complete in this sense and refer the reader to I. Namioka's paper [7] for the definition. Theorem 2 is an immediate consequence of Theorem 2.3 in [7] and generalizes Mitchell's Theorem 7 in [6] and Theorem 6 in [4] (the latter of which is a mild improvement of Rao's Theorem 2 in [8]). The full continuity of the group operations are not required for the proof and, in fact, the conclusion of Theorem 2 holds for semitopological groups. (We note that Theorem 2 could also be proved using [7; Theorem 3.1] and Mitchell's method of proof in the locally compact case [6].)

TheOREm 2. Let $G$ be a topological group and suppose that, as a topological space, $G$ is strongly countably complete and regular. Then $M(G)=L U C(G)=\left\{f \in C(G) \mid\right.$ the function $s \rightarrow l_{s} f$ from $G$ into. $C(G)$ is norm-continuous $\}$.

2. The counterexample. The following example shows that some completeness hypothesis is necessary for the conclusion of Theorem 2 to hold. 
EXAMPLE. Let $G$ be a dense countable subgroup of the usual additive real numbers $R$ with the property that every finitely generated subgroup of $G$ is in fact singly generated. ( $G$ could be the dyadic or ordinary rationals.) We construct a function $f \in C(R)$ whose restriction to $G$ is in $\mathscr{M}(G) \backslash L U C(G)$. Take $a \in R, a>0$. Let $g$ be a uniformly continuous function on $R$ with the properties

(i) $g(t)=0$ for $t \leqq 0$;

(ii) $g(n a)=0$ for all positive integers $n$;

(iii) there is $\delta>0$ such that for each $n$ there is an interval $I_{n} \subseteq[n a,(n+1) a]$ of length $\delta$ with $|g(t)| \geqq 1$ for $t \in I_{n}$.

Next, let $\left\{u_{n}\right\}$ be a sequence in $G$ which decreases to zero, is such that $u_{n}$ generates the same subgroup of $G$ as $\left\{u_{1}, \cdots, u_{n}\right\}$, and such that $\left\{u_{n} \mid n=1,2, \cdots\right\}$ generates $G$. (For example, if $G$ is the dyadic rationals, $\left\{u_{n}\right\}$ could be $\left\{1 / 2^{n}\right\}$; if $G$ is the rationals, $\left\{u_{n}\right\}$ could be $\{1 / n !\}$.) Let $H$ be any nonconstant continuous function on $[0,1]$ with $H(0)=H(1)$, and define $h: R \rightarrow R$ by

$$
h(t)=H\left(\left(t \bmod u_{n}\right) / u_{n}\right) \text { if } \quad n a \leqq t<(n+1) a .
$$

We put $f(t)=g(t) h(t)$ for $t \in R$.

The function $h$ is continuous on $R$ except possibly at the points $n a$ ( $n=1,2,3, \cdots)$; since $g$ vanishes at these points, $f$ is continuous.

The oscillation of $h$ in any subinterval of $[n a,(n+1) a]$ of length $u_{n}$ is equal to the oscillation of $H$ in $[0,1]$. If $u_{n} \leqq \delta$, the oscillation of $f$ in some interval of length $u_{n}$ is at least this, by property (iii) of $g$. Since $u_{n} \rightarrow 0, f$ is not uniformly continuous.

We use the criterion (iv) of Theorem 1 to show that the restriction of $f$ to $G$ is in $M(G)$. Suppose $\left\{s_{m}\right\}$ and $\left\{t_{n}\right\}$ are sequences in $G$ with $\left\{s_{m}\right\}$ relatively compact in $G$. Suppose

$$
\lim _{n} \lim _{m} f\left(s_{m}+t_{n}\right) \text { and } \lim _{m} \lim _{n} f\left(s_{m}+t_{n}\right)
$$

both exist. We must show they are equal. Without loss, we may assume that $s_{m} \rightarrow s \in G$, and that $t_{n} \rightarrow+\infty$. (The cases where $\left\{t_{n}\right\}$ converges to $t \in R$ or to $-\infty$ are easily dealt with.) Since $g \in \mathcal{M}(R)$, we may assume

$$
\lim _{m} \lim _{n} g\left(s_{m}+t_{n}\right)=\lim _{n} \lim _{m} g\left(s_{m}+t_{n}\right)
$$

Since $s_{m} \rightarrow s$, this last limit equals $\lim _{n} g\left(s+t_{n}\right)$, i.e., 


$$
\lim _{n} g\left(s_{m}+t_{n}\right) \underset{m}{\rightarrow} \lim _{n} g\left(s+t_{n}\right)
$$

Finally, we may assume $\left(s+t_{n}\right) \bmod a \rightarrow b$ (say), and have two cases to deal with.

(1) If $b=0$ or $a$, then by property (ii) of $g$ and uniform continuity, $\lim _{n} g\left(s+t_{n}\right)=0$; this implies that the first of the limits ${ }^{*}$ is 0 , since $h$ is bounded. Similarly, we see that the second limit of ${ }^{*}$ is also 0 .

(2) If $b \neq 0$ or $a$, we consider $m$ large enough so that $0<$ $\left(s_{m}-s\right)+b<a$, and then also, for all large enough $n$, if $k a<s+t_{n}<$ $(k+1) a$ for some integer $k$, in addition

$$
k a<\left(s_{m}-s\right)+\left(s+t_{n}\right)<(k+1) a .
$$

In the interval $[k a,(k+1) a], h$ behaves like a function of period $u_{k}$, so that, if $\left(s_{m}-s\right)$ is in the group generated by $u_{k}$ (which it will be if $k$ is large enough, and hence if $n$ is large enough),

$$
h\left(s_{m}+t_{n}\right)=h\left(\left(s_{m}-s\right)+\left(s+t_{n}\right)\right)=h\left(s+t_{n}\right) .
$$

Thus, the second of the limits * equals $\lim _{n} g\left(s+t_{n}\right) h\left(s+t_{n}\right)$, which is the same as the first limit of *.

Remarks. (a) A subgroup of $R$ generated by one rational and one irrational does not have the property we required of $G$. Whether an example exists in this case we do not know.

(b) It is no accident that the function in $\mathcal{M}(G) \backslash L U C(G)$ has an extension in $C(R)$; all functions in $\mathcal{M}(G)$ have extensions in $C(R)$. (See [3; Lemma 4.5]; a more general result is proved in [5].) What may be a little surprising is that there is a function in $\mathcal{M}(G)$ whose continuous extension to $R$ is not in $M(R)$. For, every function almost periodic, resp. weakly almost periodic, on $G$ extends to a function in $C(R)$ that is almost periodic, resp. weakly almost periodic. (See [5]. This result also holds more generally.)

\section{REFERENCES}

1. J. W. Baker and R. J. Butcher, The Stone-Čech compactification of a topological semigroup, Proc. Camb. Phil. Soc., 80 (1976), 103-107.

2. A. Grothendieck, Critères de compacité dans les espaces fonctionnels généraux, American J. Math., 75 (1952), 168-186.

3 P. Milnes, Compactifications of semitopological semigroups, J. Australian Math. Soc., 15 (1973), 488-503.

4. - Extension of continuous functions on topological semigroups, Pacific J. Math., 58 (1975), 
5. —_ An extension theorem for functions on semigroups, Proc. Amer. Math. Soc., 55 (1976), $152-154$.

6. T. Mitchell, Topological semigroups and fixed points, Illinois J. Math., 14 (1970), 630-641.

7. I. Namioka, Separate continuity and joint continuity, Pacific J. Math., 51 (1974), 515-531.

8. C. R. Rao, Invariant means on spaces of continuous or measurable functions, Trans. Amer. Math. Soc., 114 (1965), 187-196.

Received March 12, 1976. Research by the first author was supported in part by N.R.C. grant number A7857.

The University of Western ONTARio

AND

The UNIVERSITY OF SHEFFIELD 




\section{Pacific Journal of Mathematics}

\section{Vol. 66, No. $1 \quad$ November, 1976}

Helen Elizabeth. Adams, Factorization-prime ideals in integral domains ............ Patrick Robert Ahern and Robert Bruce Schneider, The boundary behavior of Henkin's kernel.

Daniel D. Anderson, Jacob R. Matijevic and Warren Douglas Nichols, The Krull

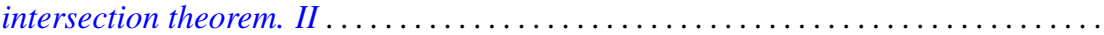

Efraim Pacillas Armendariz, On semiprime P.I.-algebras over commutative regular

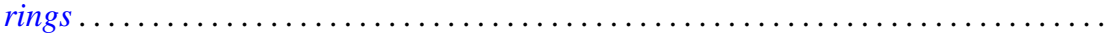

Robert H. Bird and Charles John Parry, Integral bases for bicyclic biquadratic fields

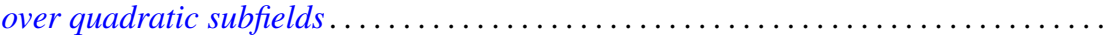

Tae Ho Choe and Young Hee Hong, Extensions of completely regular ordered

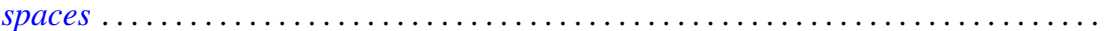

John Dauns, Generalized monoform and quasi injective modules ...............

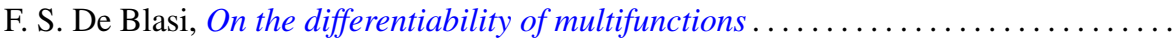

Paul M. Eakin, Jr. and Avinash Madhav Sathaye, R-endomorphisms of $R[[X]]$ are

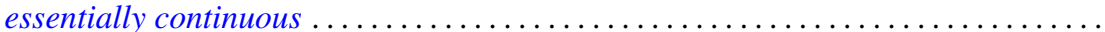

Larry Quin Eifler, Open mapping theorems for probability measures on metric

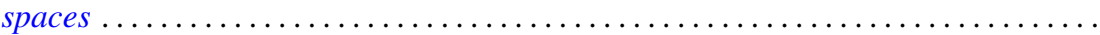

Garret J. Etgen and James Pawlowski, Oscillation criteria for second order self adjoint

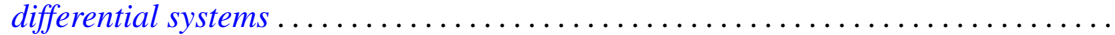

Ronald Fintushel, Local $S^{1}$ actions on 3-manifolds .

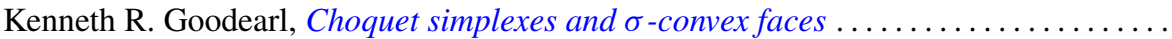

John R. Graef, Some nonoscillation criteria for higher order nonlinear differential

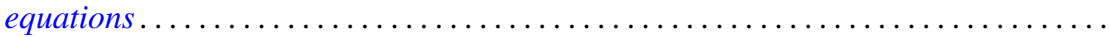

Charles Henry Heiberg, Norms of powers of absolutely convergent Fourier series: an example.

Les Andrew Karlovitz, Existence of fixed points of nonexpansive mappings in a space

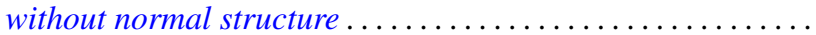

Gangaram S. Ladde, Systems of functional differential inequalities and functional

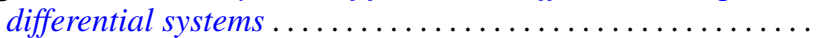

Joseph Michael Lambert, Conditions for simultaneous approximation and interpolation with norm preservation in $C[a, b]$.

Ernest Paul Lane, Insertion of a continuous function.

Robert F. Lax, Weierstrass points of products of Riemann surfaces .

Dan McCord, An estimate of the Nielsen number and an example concerning the Lefschetz fixed point theorem...

Paul Milnes and John Sydney Pym, Counterexample in the theory of continuous functions on topological groups...

Peter Johanna I. M. De Paepe, Homomorphism spaces of algebras of holomorphic functions

Judith Ann Palagallo, A representation of additive functionals on $L^{p}$-spaces,

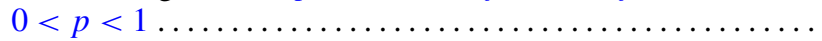

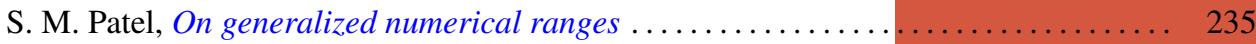

Thomas Thornton Read, A limit-point criterion for expressions with oscillatory

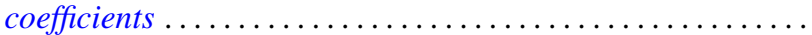

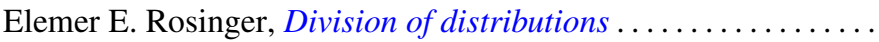

Peter S. Shoenfeld, Highly proximal and generalized almost finite

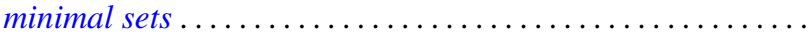

R. Sirois-Dumais and Stephen Willard, Quotient-universal sequential spaces

Robert Charles Thompson, Convex and concave functions of singular values of matrix sums....

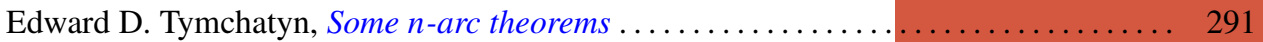

